\title{
Pontus de Tyard, poète lyrique
}

\author{
EVA KUSHNER
}

La production lyrique de Pontus de Tyard paraît minime si on la compare à celle d'un Ronsard ou d'un Du Bellay. Le petit recueil intitulé Le livre de vers liriques en contient la quasi-totalité, à moins que l'on ne considère comme faisant partie du genre lyrique les "chants" et "chansons" qui jalonnent les Erreurs amoureuses. Alors que, dans ses sonnets, Tyard poursuit inlassablement une forme et une thématique issues de Pétrarque, et sous-tendues par une vision platonicienne, ses chansons retentissent d'accents plus personnels, libérés par rapport à ses modèles tant antiques que modernes. Le chant strophique joue, dans les Erreurs amoureuses, un rôle complémentaire à celui des sonnets, et par là même fondamental. Mais les chants et chansons qui y sont insérés n'en participent pas moins à l'unité fondamentale de l'oeuvre.

Le livre de vers liriques, au contraire, donne au premier abord l'impression d'une mosaïque, car il contient visiblement les pièces n'ayant pu être intégrées aux Erreurs soit pour des raisons thématiques, soit pour des raisons de prosodie. La plupart des pièces du recueil paraissent d'abord à la suite de l'édition originale du Solitaire premier (1552). C'est en 1555 qu'est publié Le livre de vers liriques complet, à la suite de la réćdition des deux premiers livres des Erreurs amoureuses, chez Jean de Tournes, qui contient également une "tierce partie," rebaptisée Troisième livre lors de l'édition de 1573. Quelles que soient les raisons qui ont incité Tyard à grouper sous la rubrique de "vers liriques" des poèmes d'une diversité jusque là inusitée chez lui, on ne peut que noter que cette diversité s'accorde pleinement avec celle qui caractérise les Quatre premiers livres des odes de Ronsard (1550) ainsi que les Vers liriques et le Recueil de poésie de Du Bellay, datant tous les deux de 1549, donc légèrement antérieurs au Livre de vers liriques de Tyard. On s'aperçoit très vite, en outre, de ce que chaque pièce du recueil a une raison d'être spécifique liée aux prises de position de Tyard dans l'élaboration 
même du genre lyrique; si bien que ce qui se présentait au premier abord comme une mosaïque assume une unité provenant précisément de la réflexion de Tyard sur la poésie.

La vocation poétique est le motif unissant les deux premiers poèmes, "Chant en faveur de quelques excellens Poetes de ce tems" et "Chant à son Leut." Le premier poème figure d'ailleurs à la suite des la Continuation des Erreurs amoureuses (1551). Parmi les poèmes suivants plusieurs portent le nom d' "Odes." Les quatre premières célèbrent diverses divinités, à commencer par celle qui s'adresse au Ciel, afin qu'il fléchisse le coeur de sa dame. L'ode II, “Au jour des Bacchanales," célèbre Bacchus et l'esprit bachique. L'ode III, "Du socratique," loue Socrate et Platon par opposition à des philosophes plus matérialistes. La juxtaposition de ces deux odes montre bien à quel point l'ode varie du point de vue de sa gravité, apparente du moins. Quant à l'ode IV, “De ses affections," elle invoque la Muse Erato, capable d'inspirer au poète des vers capables de surmonter la mort... et l'indifférence de Pasithée.

L'ensemble des six poèmes qui viennent d'être mentionnés est consacré à la célébration des réalités les plus nobles. Les poèmes suivants chantent, ou pleurent, des réalités et des circonstances plus familières: mort de la petite chienne de Jane de Salle, mort d'un cousin, île de Pontus ou roses de cette île. Le dernier groupe comprend deux fables animales et une énigme. De cet arrangment il ressort que les premiers poèmes publiés, qui sont aussi les premiers dans l'ordre du recueil, expriment encore les soucis quotidiens de l'amant pétrarquiste et une vision platonicienne; tandis que les suivants, publiés en 1555 , se tournent plutôt vers autrui, vers la nature et le monde animal, fût-ce sur un mode mythologique. L'évolution de la poésie lyrique de Tyard serait ainsi parallc̀le à celle des Erreurs amoureuses: au moment du Troisième livre on constate, en effet, une certaine libération poétique et psychique par rapport aux deux premiers livres. Plus lentement que Ronsard, et très discrètement, Tyard ćvolue vers une révélation plus spontanée et plus naturelle du moi.

Le "Chant en faveur de quelques excellens Poëtes de ce tems" exalte la poésie et sa place dans l'ordre universel. Il cst contemporain du Solitaire premier dont il reflète les thèmes essenticls, notamment le rôle salvateur de la poésie dans la vie humaine, au sein de l'inconstance du monde. Thème de l'immortalité également, au sens que lui donne la Renaissance: le poète confère l'immortalité à celui qu'il chante, dans le même temps qu'il la conquiert pour lui-même par son chant. Poètes et rois mènent ensemble le 
combat contre l'ignorance.... Le message du poème lyrique est donc beaucoup plus direct et engagé que celui du dialogue philosophique. Dans le "Chant," Tyard compare les mérites respectifs de François ler qui a donné à la "vraie" poésie sa première chance, et de Henri II, qui préside maintenant à son développement.

Mais ce qui surtout importe dans ce poème, c'est la procession triomphale des poètes préférés de Tyard; celui-ci utilise en effet son premier poème officiellement lyrique pour déclarer ses idées en matière de lyrisme. A première vue, la succession des noms est liée à la chronologie: en gros, deux groupes de poètes, ceux qui ayant précédé Ronsard appartiennent au règne de François ler, et les contemporains de Tyard actifs sous le règne d'Henri II. Contrairement à ce que l'on pourrait attendre de la part d'un poète envers qui la fidélité de Ronsard ne se dément jamais, et dont le nom reste toujours associé à la Pléiade, Tyard relativise la place de Ronsard et de Du Bellay. C'est Saint-Gelais qui ouvre la procession. Il est loué pour "la douceur distillée/ De sa plume emmiellée" qui a su charmer deux souverains. ${ }^{1}$ C'est à Scève que s'adresse l'éloge le plus éloquent:

SCEVE si haut son sonna

Sur l'une et l'autre riviere

Qu'avec son mont Forviere

La France s'en etonna. ${ }^{2}$

Tyard se refuse à flatter Ronsard, en dépit de la rapide ascension de celui-ci. C'est Scève, que Tyard reconnaît pour son maître, qui selon lui a redonné vie à la poésie française. Après lui sont évoqués, en une même strophe, trois poètes de tradition française que Ronsard ne reconnaît pas pour siens: Héroët, Lancelot de Carle et Hugues Salel. Héroët est gratifié d'un jeu de mots flatteur portant sur son nom:

Voyez encores l'Amour,

Qui heroiquement parle

Souz Heroët... 3

L'éloge de Carle est d'un vague extrême: Tyard l'envoie séjourner au mont Hélicon, mais sans louer ses qualités poétiques alors que les trois poètes précédents, Scève, Héroët et Mellin de Saint-Gelais, font l'objet d'éloges se rapportant expressément à leurs vers. Quant à Salel, c'est en tant que traducteur qu'il est mentionné, de même que Des Masures, Marot et Martin. Tyard voulait-il ainsi amoindrir le rôle de Des Masures parce que celui-ci 
était protestant, et de Marot pour ses affinités évangéliques? Ou bien ce groupement doit-il être interprété comme élogieux, vu le prestige dont la Deffence et illustration venait de revêtir la traduction? Toujours est-il que le fait de réunir les traducteurs nuance l'éloge qui leur est adressé, mettant en valeur d'une part la strophe consacrée au seul Scève, et d'autre part celle qui suit et que se partagent Ronsard et Du Bellay:
Osera quelqu'un celer
L'honneur de ta main, qui guide
L'immortalisante bride
Du cheval nouant ${ }^{4}$ par l'air,
Ronsard? Tu t'es pu vanter,
En François fredons liriques,
Prince des neuf Grecs antiques:
Pendant que le tret puissant
De l'aveugle esblouissant,
Feit si bien Bellay chanter
Son rameau verpalissant. ${ }^{5}$

Ici, Tyard commence par défier les détracteurs imaginaires (ou réels) de Ronsard; puis il cite le titre de gloire que Ronsard s'attribue lui-même (et que répète Du Bellay dans "Contre les poètes envieux," en 1550). Ni l'un ni l'autre élément éiogieux n'est donc assumé résolument par Tyard lui-même; or, il s'agit du genre lyrique, dans lequel Tyard est précisément en train de s'exprimer pour la première fois. Dans le cas de Du Bellay au contraire, même s'il est pour Tyard, au moment précis où le "Chant" est écrit, un concurrent puissant en fait de poésie amoureuse, Tyard dit sans réserve son admiration pour l'Olive.

C'est Des Autels qui clôt la procession triomphale: il incarne l'avenir de la poésie, aux yeux de Tyard du moins, dont la fidélité vis-à-vis de son cousin fut inépuisable - alors que Ronsard et Du Bellay en sont les gloires présentes et que les poètes du premier groupe s'étaient surtout illustrés dans le passé. Tel est donc le Panthéon poétique de Tyard. En conclusion, celui-ci revient aux "longs pensers ardens/ Sans cesse en moy residens"6 et à ses "peines languissantes"; mais non sans avoir parlć poćtiquement de la poćsie dont l'amour y devient consubstanticl à celui de la bien-aimée.

Il est clair que la liste des poètes célébrés ne correspond pas à celle de la Pléiade, mais manifeste les préférences esthétiques de Tyard lui-même, qui sont aussi celles de Des Autels. En comparaison, la liste figurant dans la Musagnoeomachie de Du Bellay réconcilie généreusement passé et présent 
de la poésie en France ou plutôt, puisque les lignes de partage ne sont pas uniquement chronologiques, fait place aux tendances traditionnelles aussi bien qu'à celles instaurées par le groupe de Ronsard. Sont nommés: Carle, Héroët, Saint-Gelais, "les trois favoriz des Grâces,"7 ainsi que Scève et Salel; "l'utiledoux Rabelais," Bouju, Peletier, Maclou, Macrin, Tyard, Pascal; enfin, "trois flambeaux," Baï, Dorat, et en tout dernier lieu Ronsard, ce qui prouve bien que le sommet de la gloire appartient à celui qui clôt ce type de cortège poétique.

Chez Ronsard, si conscient de son rôle parmi les poètes de sa génération, ce genre de liste possède une valeur particulièrement symbolique: pour lui, nommer quelqu'un, c'est l'inclure parmi les siens. Que ce soit dans les "Dithyrambes a la pompe du bouc de E. Jodelle, poete tragiq'," le "Voyage d'Hercueil," ou encore "Les Isles fortunées," n'apparaissent en général que les noms de poètes ou érudits participant au renouvellement des lettres et des idées selon sa propre optique: et ceux de quelques amis de jeunesse; d'autant plus lorsqu'il s'agit de constituer la Pléiade, dans des poèmes tels que "L'élegie à Jean de la Péruse" (1553), "L’hymne de Henry II" dans sa version de 1554 et le même hymne dans sa révision de 1584 .

$\mathrm{Du}$ Bellay, ainsi que nous venons de le noter, s'associe dans la "Musagnoeomachie" quelques aînés absents du chapitre "Des poètes françoys" de la Deffence et Illustration. Là, en effet, à la suite de Jean Meung et Guillaume de Lorris, seul Lemaire de Belges est directement nommé. Le nom de Marot apparaît comme celui de l'auteur d'une épigramme à Salel distinguant un certain nombre de poètes qui ne sont autres que les Grands Rhétoriqueurs. Du Bellay n'intègre pas l'épigramme à son texte; c'est le lecteur qui doit la rechercher s'il veut en savoir davantage sur ces poètes d'antan.

C'est dire que les sympathies poétiques de Tyard se distinguent assez fortement de celles de Ronsard et Du Bellay, surtout en ce qu'il accueille chaque poète, traditionnel ou novateur, à titre individuel plutôt qu'en fonction d'une allégeance collective. Le "Chant en faveur de quelques excellens Poëtes de ce tems" ne parle ni de la Brigade ni de la Pléiade, métaphores qui ne semblent avoir pour Tyard aucune réalité. Il ne prend pas non plus parti pour Barthelemy Ancau qui venait de riposter aux Quatre premiers livres des odes eil déniant à Ronsard la nouveauté que celui-ci revendiquait tant. Le "Chant" se situe, sinon au-dessus, du moins en dehors de ia mêlée. C'est Guillaume des Autels qui s'était chargé de défendre les thèses du Quintil Horatian en 1550, dans la préface de son Repos de plus 
grand travail ainsi que dans la Réplique aux furieuses defenses de Louis Meigret. La question de savoir si l'ode est véritablement un genre nouveau devient l'enjeu d'une dispute entre Ronsard et Saint-Gelais devant la Cour, et, du coup, celui de l'affrontement entre tradition et innovation. Grand seigneur, Tyard réagit avec une indépendance nuancée. Sans déclarations théoriques, il s'essaie à son tour dans le genre lyrique où, vu les circonstances, chaque poème équivaudra à un choix théorique. Le poème que nous venons d'analyser s'intitule "Chant," mais plusieurs des suivants sont nommés "Odes," ce qui signifie qu'avec Des Autels Tyard croit les deux appellations interchangeables et qu'il considère qu'il y a continuité au sein du genre lyrique dans l'histoire récente de la poésie en France. N'accueille-t-il pas, d'autre part, Mellin de Saint-Gelais parmi les créateurs? Le moins que l'on puisse dire, c'est que tout ceci va à l'encontre de la notion d'une Pléiade monolithique dont Tyard serait "membre."

Cela n'empêche pas - au contraire - qu'une forte intertextualité ait alors existé entre Ronsard, Du Bellay et Tyard. Ainsi, le thème de l'inconstance sur lequel s'ouvre le "Chant en faveur de quelques excellens Poëtes de ce tems" fait écho à deux au moins des poèmes lyriques de Du Bellay: "Des miseres et fortunes humaines," et "De l'inconstance des choses." Dans les "Vers lyriques" de Du Bellay, d'autre part, l'ode "De l'immortalité des Poctes" développe longuement ce que Tyard dit en quelques vers au sujet de la survie poétique.

Semblablement, le "Chant à son Leut" rappelle par son titre plusieurs poèmes de Ronsard adressés à un instrument musical. "A sa lyre," "A sa guiterre" et "A son lut" datent tous les trois de 1550; le poc̀me de Tyard leur rćpond. Dans le "Chant en faveur de quelques excellens Poctes de ce tems," Tyard rappelle les "François fredons liriques" dans lesquels Ronsard se vante de l'importance de son "invention" de l'ode en France. Tyard continue ensuite à lui répondre par le "Chant à son Leut"; en suivant de très près le titre du poème ronsardien "A sa lyre" il n'en attire que davantage l'attention du lecteur sur ce qui le sépare de Ronsard. Le Chant est écrit en "terze rime," forme italienne que Tyard affectionne, mais que n'emploic pas Ronsard. Dans le poème de Ronsard, la lyre est symbole de l'état de la poésic en France, vis-à-vis de laquelle il est lui-même le principal intervenant. Chez Tyard, l'accent est vraiment sur la poésic elle-même: le luth est personnifié et c'est à lui que, sous la forme d'une suite d'impératifs "Chante, mon Leuth..." - Tyard confic son propre chant d'amour. Mais il s'agit bien d'une réflexion sur la forme du poème, et sur les discussions 
de l'heure à propos de la poésie. Tyard se résout à délaisser sa "mortelle plainte" pétrarquiste pour entonner un chant "plus doux"8 à l'instar de Saint-Gelais, mentionné une fois de plus dans ce contexte, donc avec l'intention de l'inclure parmi les influences fastes. Le chant lyrique sera un chant de célébration plutôt que de plainte. Comme Ronsard et ses amis, Tyard profitera de la liberté de mètre et de rythme offerte par l'ode, de son régime strophique, de son ouverture à l'imagerie et à la mythologie antiques. L'ode lui servira, en particulier, à célébrer une fois de plus la beauté de Pasithée, mais en abandonnant la mélancolie des Erreurs et en adoptant une certain joie sensuelle, inusitée sous sa plume. Donc, tout, dans Le livre de vers liriques, y compris les vers amoureux, concourt à en faire un recueil conçu en vue de diverger par rapport aux Erreurs amoureuses.

En particulier, le "Chant à son Leuth" est dominé par une intense conscience esthétique, résolue à dire la nature du chant au travers même de la poésie amoureuse. Pasithée demeure inaccessible; c'est le regard poétique qui la perçoit d'une manière différente. Dépouillée du voile du sacré, elle paraît plus proche et plus humaine:

Chante ces cent et cent graces semées

Parmi ce ris, ris chastement folastre:

Qui tient en moy cent torches allumées. ${ }^{9}$

Par sa vie et sa richesse sensuelles ce poème rejoint donc l'ode légère ronsardienne de cette époque (du type "Ma petite columbelle") en attendant qu'à son tour Ronsard rejoigne Tyard, dans les Amours de 1552 et 1553, par la manière dont il y unit la beauté physique à la beauté morale. En un sens, le "Chant à son Leuth" se lit comme un blason du corps féminin; mais la louange de ce corps est inséparable de celle de l'âme ardente et raffinée qui l'habite; et de l'appartenance ultime de cette âme:

Mais change moy celle immortelle

Qui, pour tenter du Ciel nouvelle trace,

Son aesle empenne, et son vol renouvelle. ${ }^{10}$

Si le "leuth" de Tyard ne possède pas, de son propre aveu, assez de puissance pour capter la beauté elle-même, il peut du moins continuer à parler au coeur de Pasithée. La grandeur du projet de Tyard réside en sa modestie même: il consiste à démontrer ce que Des Autels déclare théoriquement, à savoir la continuité réelle de la tradition lyrique en France, qui apparaît dès que l'on accepte la co-existence, tant dans les ocuvres des 
"marotiques" que dans celles de Ronsard et de ses disciples, de l'ode grave et de l'ode légère: le Livre de vers liriques en constitue précisément un exemple, et Tyard exploite pleinement cette exemplarité.

A la suite des années 1549-51 dont il est question ici, les enjeux changeront rapidement: dans l'Art poetique françoys de Peletier du Mans (1555), parlant au nom d'une Pléiade mûrie, la continuité entre la chanson marotique et l'ode ronsardienne est rétablie, Ronsard et Saint-Gelais se réconcilient, et le "style bas" adopté par Ronsard réduit à néant l'opposition des deux "écoles."

Les deux poèmes programmatiques que nous venons de commenter sont suivis, dans le Livre de vers liriques, d'une séquence de poèmes de louanges, à commencer par l' "Ode premiere au ciel en faveur de sa Dame." La forme en est celle d'un voeu: le poète invoque sur sa dame la faveur du Ciel, liant amour et poésie dans le symbole de la flamme qui brûle son âme. A chaque don que possède la dame, le Ciel est invité à répondre en l'éternisant; jusqu'à la septième strophe. Si le poème s'arrêtait là, il se rattacherait, thématiquement, aux Erreurs amoureuses; mais il continue, transformant l'intemporalité en éternelle jeunesse, que le poète contemple avec passion et douceur. La convention pétrarquiste ne permettait pas, dans les Erreurs, une simple victoire de l'amour humain; ici, le théâtre de l'écriture lyrique rend possible une vision triomphante de l'amour: donc également de la poésie, comme représentation.

Lors les vers que je feray

Richement j'estofferay,

En louange immortelle

De toy, et d'elle. ${ }^{11}$

L'ode "au jour des Bacchanales" constitue un nouvel exemple de création parallèle entre Ronsard, Du Bellay et Tyard. Dans les Vers lyriques de Du Bellay figure l'ode "Du jour des Bacchanales," hommage et voeu adressé à Bacchus; ce n'est qu'à la fin du poème que Du Bellay tire de l'agitation bachique une morale déjà présente chez Horace, c'est-à-dire un éloge de la folic: "Quelquesfois il faut faire/ Le fol pour son amy."12

De son côté, Ronsard dans le "Chant de folie à Bacchus"13 crée un tableau mythologique pittoresque, sonore et mouvementé, qui semble n'exister que pour le plaisir du lecteur et ne solliciter d'autre réaction que l'insouciance. "L'Hymne de Bacchus" de 1555 ajoute à la description de Bacchus tous les souvenirs mythologiques liés à lui et évoquera son pouvoir 
universel, sans oublier son rôle dans l'éveil de l'inspiration poétique: "Par toy, Pere, chargez de ta douce ambrosie/ Nous elevons au ciel l'humaine fantaisie." 14

En comparaison, ce qui distingue le poème de Tyard, c'est que c'est le poète plutôt que le dieu qui en est l'acteur principal. Tyard commence par prendre à son compte, avec une allégresse rare chez lui, l'insouciance qu'exige le genre. Il se livre à ce que l'on appellerait maintenant la carnavalisation. Il se libère de toute contrainte, y compris celle de l'amour! Ses Muses titubent d'ivresse et, saisi de frénésie, il s'attribue une virilité agressive. Cette ode forme, en attendant les Douze fleuves ou fontaines, l'envers dionysiaque des Erreurs amoureuses et du Solitaire premier, remplissant un profond besoin de l'imaginaire chez ce poète qui, n'ayant pas écrit de Folastries, n'en tenait pas moins à manifester, à l'occasion, une verve truculente. Mais il serait erroné de lire "Au jour des Bacchanales" uniquement comme le signe d'une surcompensation. Le foisonnement bachique symbolise tout le contradictoire et la multiplicité du divers, et l'attrait de celui-ci pour Tyard.

Le rôle de l'ode "Du socratique," qui suite immédiatement, est-il antithétique et même contradictoire? Nous le pensons plutôt complémentaire en ce qu'il explore aussi la liberté de l'âme, mais d'une autre manière. Comme dans le Solitaire premier, Tyard exprime ici sa désapprobation vis-à-vis de l'épicurisme; mais il condamne aussi d'autres errements philosophiques et moraux, et, à la limite, toute doctrine, car il est épris uniquement de "ce, qui est pur du Ciel."15

Il ne prétend proférer aucune vérité nouvelle; c'est dans l'Age d'or du passé qu'il espère trouver un remède aux tragiques carences de son temps: hypocrisie, injustice, avidité. Si l'Age d'or revenait, "Jupiter" agréerait l'offrande la paix parmi les hommes, plutôt que des sacrifices sanglants. Irénisme, donc, mais également satire de tout ce qui, au scin de l'Eglise, prétend représenter la divinité sous des traits matéricls. Les figures mythologiques voilent à peine ici une pensée essentiellement thćologique, puisant encore son hiérarchie des valcurs dans la Theologia platonica de Ficin, tout en s'éveillant aux problèmes spiritucls de son sic̀cle. La magnifique invocation au Centre, de qui part toute beauté et en qui réside tout bien, contient, à notre avis, la figure-clef de la vision tyardiennc, active non seulement dans la poésie des années 50 mais dans toute son ocuvre subséquente. Car c'est une figure qui réconcilie - poétiquement sinon philosophiquement - la notion de l'immatérialité de l'être avec celle de la 
réalité et de la beauté de ses manifestations concrètes. Si la sphère entière du réel peut être perçue comme coextensive avec la présence du bien, alors il pourra y avoir une poésie et une philosophie chrétiennes sans culpabilité, et le Tyard de la Contre-Réforme n'aura pas à renier, mais seulement à transformer sa vision platonicienne. ${ }^{16}$

L'ode "De ses affections" termine la série des odes philosophiques et les résume toutes en une histoire poétique de l'âme humaine et de ses vicissitudes. Parce qu'il y a là une traduction en vers, très concentrée, de la première partie du Solitaire premier, on peut y discerner d'autant mieux l'art et la fonction de l'ode chez Tyard. L'âme est représentée d'une manière vivante et pathétique avec son éternelle nostalgie des cieux, et son appétit de beauté et de bonheur terrestres. Il est vrai que tout homme est écartelé entre ces deux tendances; mais être poète, c'est s'arracher au "mol troupeau des delices" pour suivre sa Muse. Le moment de la vocation poétique fait songer à celui d'une vocation religieuse. La Muse Erato le choisit pour sien, afin

[...] qu'encore l'amoureux son

Jusques en nostre Helicon

De ta douce lyre arrive. ${ }^{17}$

Tyard fait ensuite le bilan de sa propre histoirc poćtique et personnelle, et, en particulier, de son pétrarquisme:

J'ay chanté ma passion

Inconstante constamment

En glace, en feu, du tourment

Qui l'esprit me mine, et ronge. ${ }^{18}$

Histoire poétique et histoire personnelle aboutissent ensemble à un double renoncement; les contemporains savaient, eux, quel était "l’impiteux" qu'épousa Pasithéc, mais ils savaient aussi pourquoi la poésie de Pontus était si rigoureusement condamnée à être le tombeau de la "verité lapidée"19 alors que d'autres poètes s'épanouissaient en développant d'autres formes de discours. Exceptionnellement ici, grâce à la liberté qui appartient à la définition même du genre lyrique, Tyard exhale une plainte plus directe que celle qui caractérise les Erreurs amoureuses.

Les poèmes qui suivent forment un autre groupe thématique; on pourrait les classer comme "odes légères": quatre d'entre elles sont d'un caractère plus familier que le groupe précédent, deux sont des fables mythologiques 
et le tout dernier poème est une énigme. John Lapp, à qui l'on doit l'édition des Oeuvres poétiques complètes de Tyard, s'est surtout intéressé à un trait qui relie entre eux tous ces poèmes, que leur sujet soit, ou non, mythologique: à savoir, le sens de la métamorphose, signe d'esthétique baroque. Sans contester cette observation essentielle, il nous paraît important de noter qu'elle concorde avec ce que l'on sait par ailleurs ${ }^{20}$ de la réorientation de Tyard vers le concret et la diversité, aux environs de 1555 , c'est-à-dire l'année de publication du groupe de poèmes en question.

"Sur la mort de la petite chienne de Jane, nommée Flore" relate un incident survenu à une amie autre que Pasithée; et cet incident concerne un animal plutôt qu'un être humain: voilà en un même poème deux traits exceptionnels dans la poésie de Tyard jusqu'à cette date. En outre, Tyard joue très librement avec les données de la mythologie antique. Il fait revivre Zeus uniquement pour le plaisir de le voir manifester sa toute-puissance afin de consoler le chagrin d'une enfant, quitte à déranger pour elle tout l'ordre de l'univers. Ayant envoyé son aigle chercher la dépouille mortelle de la petite chienne, Jupiter s'occupe paternellement de lui trouver une place aux cieux, où elle sera transformée en constellation. Certes, ce poème comporte un aspect mondain - les salons du XVI $\mathrm{X}^{\mathrm{e}}$ siècle aimaient transformer en jeu poétique tout incident de la vie quotidienne. ${ }^{21}$ Mais plus important encore est l'effet produit sur l'imagination de Tyard par le foisonnement du divers, par la notion d'une victoire sur la mort, et par la complicité de la nature. La fin du poème rejoint en effet son commencement pour proclamer le triomphe de la poésie sur la mort...

L' "Epicede," ou "Regret à la mort de Monsieur L'Escuyer de Saint-Sarnin son cousin" est le seul chant funèbre du recueil. Il est dominé par le thème de l'inconstance, qui est aussi un des thèmes principaux des Vers lyriques de Du Bellay. A la différence de celui-ci, Tyard s'attarde peu sur la "cruauté du sort" une fois qu'il l'a fortement constatée. En revanche, un thème plus spécifique se fait jour ici, celui de l'injustice s'acharnant sur les vertueux. Saint-Sarnin fut victime d'un assassinat politique, et Tyard s'identifie au sort de cet homme qu'il admirait, lui qui, d'une autre manière, fut victime d'un "impiteux." Tandis que Saint-Sarnin vit en Dieu et dans la mémoire des hommes, Tyard traîne encore sa peine... Mais l'accès à l'immortalité poétique passe par la porte étroite de la souffrance.

Comme Ronsard, Tyard a chanté un lieu familier de son adolescence et de sa jeunesse: deux odes sont consacrées à son île. ${ }^{22}$ L' "Ode, en nom de son isle” prend la forme d'une prosopopée. L'île elle-même déclare 
appartenir “à Pasithée, et Eraton.” Elle convie à entrer ceux qui connaissent l'amour véritable, et interdit de séjour les ennemis de la poésie... C'est l'utopie personnelle de Pontus, connue de Bugnyon, qui l'appelle l'île Pontique.

Particulièrement riche en connotations, cette ode confirme la double vocation du Livre de vers liriques et de toute la poésie tyardienne: comme l'île, ils sont voués à Pasithée et Erato. Amour et poésie sont inséparables et font partie du même pari spirituel en faveur de l'idéal, et de la vision du monde qui en est le fruit. La consécration de l'île à Pasithée et Erato est énoncée, parallèlement, dans la première et dernière strophe, ce qui donne encadrement et clôture à la caractérisation de l'île.

L'île est aussi refuge par rapport à la Ville, et lieu de vie contemplative par opposition à la vie active. De même, Ronsard conviera Hélène à venir avec lui "sur le Jourdain," délaissant la Cour. Mais, contrairement aux loci amoeni des oeuvres passées de Tyard, son île n'est pas un séjour solitaire; un "autel religieux" y est dressé en l'honneur des poètes qui ont chanté leurs amours. Et, surtout, Tyard est devenu sensible à la présence de la nature, tout particulièrement de la flore, qu'il célèbre avec allégresse.

Dans l'ode "Les roses de son isle," cette présence de la nature éclate à travers une longue comparaison de la rose et de la femme. Pontus s'y penche sur le devenir des êtres et des choses, insistant, ainsi que l'a noté John Lapp ${ }^{23}$, sur les moments de passage. Son regard devient plus observateur que contemplatif; il se cherche des objets plus concrets qu'auparavant, dont son oeil détaillera le mouvement. (Parallèlement, on peut noter qu'au tournant de 1555-56, une évolution semblable se fait jour parmi ses discours en forme de dialogues).

Quoi de plus mouvementé, enfin, que les deux fables mythologiques du recueil: "L'ode au rossignol," et "A l'arondelle d'un ennuy secret" et "Les grenoilles.” Deux odes de Ronsard, retranchées en 1584, ont respectivement pour titre "A un rossignol," et "La grenouille, à Remy Bclleau." Mêmes sujets en apparence, même ordre; mais la ressemblance s'arrète là. Les deux odes de Ronsard blasonnent le rossignol et la grenouille; Tyard raconte des fables mythologiques. Dans "Les grenoilles" il suit de près Ovide à propos de l'histoire de Latone se vengeant des "vilains" qui refusent de la laisser se désaltérer. Pur exercice de style de la part du poète, sur la laideur, après avoir exalté la beauté des roses? Ou vengeance délectable de l'imagination à l'égard de tous les ingrats, voire d'une certaine ingrate? 
La manière dont Tyard traite le fable de Philomèle et de Progné tend à confirmer cette dernière notion: il y fait alterner des strophes à contenu mythologique avec des strophes le concernant personnellement, en une longue comparaison de sa propre souffrance avec celle des belles-soeurs de la fable. Métamorphosées en oiseaux, Progné et Philomèle connaissent une vie nouvelle et libre, tandis que le poète ne connaît qu'esclavage et mort; mais ils se retrouvent tous les trois dans la nature et dans le chant du poète, dont le malheur résume tous les malheurs d'autrui.

Ainsi, le Livre de vers liriques se distingue au sein de son oeuvre, et vis-à-vis des oeuvres contemporaines de la sienne, par sa variété tant métrique que thématique, sa vivacité, sa teneur en mythologie, sa réflexion sur le genre lyrique, et surtout la manière très personnelle dont Tyard orchestre tous ces éléments.

Victoria University, University of Toronto

\section{Notes}

1 Pontus de Tyard, Oeuvres poétiques complètes, éd. critique par John C. Lapp (Paris: Didier, 1966), p. 158. (Dans les notes qui suivent, cette éditon sera désignée par le sigle OPC).

2 Ibid, p. 159.

3 Ibid.

4 Nageant.

$5 O P C$, p. 160

6 Ibid., p. 161.

7 Ibid., p. 162.

8 Ibid., p. 163.

9 Ibid., p. 166.

10 Ibid., p. 164.

11 Ibid., p. 166.

12 Joachim du Bellay, Vers lyriques, éd. crit. H. Chamard (Paris: Droz, 1934), t. III, p.29.

13 Bocage de 1550.

14 Ed. crit. Laumonier, t. VI, p. 189-90.

$15 O P C$, p. 172.

16 En rétrospective, Tyard lui-même est devenu conscient de cette conversion de tout son acquis intellectuel. cf. "Summa Christus felicitas," poème liminaire des Trois livres d'Ilomilies (1586).

17 OPC., p. 178.

18 Ibid.

19 Ibid., p. 180.

20 Il est communément admis que ce changement d'orientation de Tyard coïncide avec la composition du Discours de temps, de l'an et de ses parties. Cf., par exemple, E. Kushner, "Le rôle de la temporalité dans la pensée de Pontus de Tyard," Le temps et la durée dans 
la littérature du Moyen Age et de la Renaissance, éd. Yvonne Bellenger (Paris: Nizet, 1985), p. $211-30$

21 cf. sur ce point L. Clark Keating, Studies on the Literary Salons in France (1550-1615) (Cambridge: Harvard University Press, 1941).

22 Nous avons longuement cherché cette île aux environs du château de Bissy-sur-Fley, sans succès: il n'y a plus, actuellement, de cours d'eau sur le domaine ou à proximité, ni d'ailleurs d'étang.

23 "On constate dans cette ode l'intérêt qu'éprouvait Pontus pour le moment de transition le passage d'un état à l'autre - qui fait de lui le vrai poète des métamorphoses.... Le poème consiste donc en une série de transitions: l'aube qui cède la place au soleil, telle fleur qui s'ouvre à la lumière, telle autre dont le bouton, à mesure qu'il grandit, est percé par une épine, telles autres qui changent de couleur devant les yeux du poète..." Introduction, $O P C$., p. XLI. 